\title{
The road less traveled: strategies to enhance the frequency of homology-directed repair (HDR) for increased efficiency of CRISPR/Cas-mediated transgenesis
}

\author{
Sushil Devkota ${ }^{*}$ \\ Section of Cell and Developmental Biology, University of California San Diego, La Jolla, CA 92093, USA
}

\begin{abstract}
Non-homologous end joining (NHE)), and to a lesser extent, the error-free pathway known as homology-directed repair (HDR) are cellular mechanisms for recovery from double-strand DNA breaks (DSB) induced by RNA-guided programmable nuclease CRISPR/Cas. Since NHEJ is equivalent to using a duck tape to stick two pieces of metals together, the outcome of this repair mechanism is prone to error. Any out-of-frame mutations or premature stop codons resulting from NHEJ repair mechanism are extremely handy for loss-of-function studies. Substitution of a mutation on the genome with the correct exogenous repair DNA requires coordination via an error-free HDR, for targeted transgenesis. However, several practical limitations exist in harnessing the potential of HDR to replace a faulty mutation for therapeutic purposes in all cell types and more so in somatic cells. In germ cells after the DSB, copying occurs from the homologous chromosome, which increases the chances of incorporation of exogenous DNA with some degree of homology into the genome compared with somatic cells where copying from the identical sister chromatid is always preferred. This review summarizes several strategies that have been implemented to increase the frequency of HDR with a focus on somatic cells. It also highlights the limitations of this technology in gene therapy and suggests specific solutions to circumvent those barriers. [BMB Reports 2018; 51(9): 437-443]
\end{abstract}

\section{INTRODUCTION}

The discovery of restriction enzymes enabled researchers to effectively manipulate DNA in vitro (1). The next step in this revolution was the ability to alter genomic DNA inside an organism. Collective efforts from several laboratories spanning

*Corresponding author. Tel: +1-858-999-7879; Fax: +1-858-9997879; E-mail: sdevkota@ ucsd.edu

https://doi.org/10.5483/BMBRep.2018.51.9.187

Received 8 July 2018

Keywords: CRISPR/Cas, DNA-repair, HDR, NHEJ, Therapeutics almost a decade in the 80's laid the foundation for targeted transgenesis in mammalian cells (2-4). The fundamental observation driving these studies was that mammalian cells integrate an exogenous piece of DNA in a site-specific manner if the donor DNA contains sequences with sufficient homology to the genomic DNA. Martin J. Evans, Oliver Smithies, and Mario R. Capecchi were awarded 2007 Nobel Prize in Physiology or Medicine for their roles in this discovery that ushered in an era of genome engineering (5). However, there were several practical limitations to utilizing the intrinsic cellular HDR machinery for transgenesis. First, the rate of integration of the donor DNA was low, making this process extremely labor-intensive. Furthermore, there was always a chance of random integration of the donor DNA in a non-specific manner, thus, leaving the possibility of false readouts (6). Similar to many scientific breakthroughs, the solution to this critical problem was obtained from the study in yeast by Maria Jasin and colleagues who demonstrated that introduction of site-specific breaks in the chromosome using an 18-bp meganuclease Scel enhanced the rate of locus-specific HDR (7). This discovery opened up the new field dedicated to finding new programmable meganucleases that could cover all areas of the genome. Two of the programmable nucleases that are widely used are zinc finger nucleases (ZFNs) (8) and the transcription activator-like effector nucleases (TALENs) $(9,10)$. These nucleases contain two major components: 1) DNA-binding capacity of zinc fingers or TALE transcription factors to guide the system to a specific site in the genome; and 2) Fokl endonuclease that makes a cut in the genome. However, there are several limitations to the use of ZFNs and TALENs including the necessity to redesign constructs with every new target, the larger size of the constructs, and the requirement to execute complex protein engineering for generating these systems (11). All these hurdles in the successful genome engineering were addressed in 2012 by a landmark study led by Doudna and Charpentier showing that the components of bacterial CRISPR/Cas defense system can be distilled down as a programmable nuclease and tailored to cleave a specific DNA sequence (12). At its core, the CRISPR/Cas system consists of an endonuclease whose DNA-targeting specificity is dictated

ISSN: 1976-670X (electronic edition)

Copyright (C) 2018 by the The Korean Society for Biochemistry and Molecular Biology

(c) This is an open-access article distributed under the terms of the Creative Commons Attribution Non-Commercial License (http://creativecommons.org/licenses/by-nc/4.0) which permits unrestricted non-commercial use, distribution, and reproduction in any medium, provided the original work is properly cited. 
by a short-guide RNA. A year later, Feng Zhang and George Church group reported the applicability of CRISPR/Cas9 to edit mammalian cells (13). Since then, the simplicity and efficiency of CRISPR/Cas system has established it as a household tool in research labs throughout the world. It is amazing that a bacterial defense mechanism designed to degrade bacteriophage DNA as a protective mechanism and had probably never been exposed to a nucleosome-bound chromosome in a natural setting, cleaves with such precision and efficiency in mammalian cells. Beyond the endonuclease activity, different versions of Cas9 such as enzymatically dead Cas9 (dCas9) can be used for gene regulation by fusing with transcriptional activators and suppressors, epigenetic regulation by targeting histone modifying enzymes, and manipulation of chromatin topology (14).

Double-strand breaks (DSBs) are unavoidable consequences accompanying cellular life. Living organisms have evolved elaborate repair machineries to fix DSBs whether the source of the break is natural or experimentally induced by Cas9. DSB repair mechanisms are grouped under two major categories: Non-Homologous End Joining (NHEJ) and Homology-directed Repair (HDR) (15). The choice of NHEJ versus HDR as a preferred repair machinery is not arbitrary and several factors contribute to the decision-making. Strategies to manipulate either of these repair mechanisms can affect the outcome of gene editing and should be considered carefully before proceeding to experiment. Resembling the scenario in classical Robert Frost's poem, "The Road Not Taken", HDR is the less traveled (less prevalent) form of repair; however, proper utilization of this repair strategy for targeted transgenesis is bound to "make all the difference".

[The last stanza in Robert Frost poem reads:

I shall be telling this with a sigh

Somewhere ages and ages hence:

Two roads diverged in a wood, and I-

I took the one less traveled by,

And that has made all the difference.]

The current review focuses on several strategies that have been implemented to increase the frequency of HDR in somatic cells and provides a perspective on the challenges that lie ahead for potential application of this technology for gene therapy in humans.

\section{NHEJ VS. HDR}

After the generation of DSBs by Cas9, they need to recognized and repaired by evolutionarily conserved cellular DNA repair mechanisms namely NHEJ and HDR. NHEJ is the predominant form of mammalian DNA repair mechanism that successfully joins broken pieces of DNA together (16). Based on the molecular players involved, NHEJ pathway is further divided into canonical non-homologous end joining (c-NHEJ) and alternative non-homologous end joining (alt-NHEJ) also referred to as microhomology-mediated end-joining (MMEJ). The major molecular players in c-NHEJ are ku70/ku80 heterodimer and DNA protein kinase catalytic subunit (DNA-PKcs) (17). The KU70/KU80 heterodimer binds to DSBs along with DNA-PKcs. This molecular assembly protects the DNA wound site and maintains the ends in close proximity, which is critical for rejoining the pieces of DNA together. The auto kinase activity of DNA-PKCs or transphosphorylation by ataxia telangiectasia mutated (ATM) kinase results in the recruitment of Artemis and DNA pol $\lambda$ and $\mu$ to the target cleavage site (18). Artemis acts as a $5^{\prime}$ exonuclease to generate $3^{\prime}$ single-stranded DNA (ssDNA) overhangs that are further processed to create blunt DNA ends. The blunt ends are then ligated by the action of $\mathrm{X}$-ray repair cross-complementing protein 4 (XRCC4), XRCC4-like factor (XLF), and DNA ligase 4 (LIG4) to complete the repair process (19).

Alt-NHEJ pathway works independently of KU70/KU80 resulting in larger deletions and chromosome translocations (20). The major molecular player in this form of repair is poly [ADP-ribose] polymerase 1 (PARP1) that competes with KU70/KU80 for binding to DNA ends and thus is refractory to the c-NHEJ. PARP1 facilitates stabilization of $\gamma \mathrm{H} 2 \mathrm{AX}$ (phosphorylated form of $\mathrm{H} 2 \mathrm{AX}$ ) via its ADP-ribosylation activity on nucleosome exchange factor SUPT16H and results in the formation of MRE11-Rad50-NBS1 (MRN) complex tilting the balance of repair machinery away from c-NHEJ in favor of alt-NHEJ (21). Mechanistically, alt-NHEJ resembles HDR because it is favored in the $\mathrm{S}$ and $\mathrm{G} 2$ phases of cell cycle unlike c-NHEJ that is active in all stages of mitosis. Moreover, the choice of DNA ligase in c-NHEJ is LIG4, whereas in alt-NHEJ, LIG1 and 3 are utilized (22).

Accuracy of DNA repair is strongly enhanced by the utilization of the sequence from the sister chromatid or homologous chromosome, and constitutes the basis of HDR. Since sister chromatids are available at the S/G2, HDR is restricted to these phases of cell cycle (23). The first step in the HDR pathway is phosphorylation of $\mathrm{H} 2 \mathrm{AX}$ by ataxiatelangiectasia mutated (ATM) and ataxia-telangiectasia and Rad3-related protein (ATR) in conjunction with the mediator of DNA damage checkpoint 1 (MDC1) that results in the localized accumulation of $\gamma \mathrm{H} 2 \mathrm{AX}$ at the DNA damage site (24). Next, the MRN complex localizes to the DSB and plays a stabilizing role to inhibit chromosomal breaks. Following the stabilization of the initial DSB, the $5^{\prime}$ exonuclease activity by either MRE11/CtIP (short resection) or Exo1/BLM (long resection) results in the generation of $3^{\prime}$ single strand ( $\left.3^{\prime} \mathrm{SS}\right)$ overhangs that are covered by human replication protein $A$ (RPA) (25). Rad51 in conjunction with breast cancer 1 and 2 (BRCA1 and 2) along with partner and localizer of BRCA2 (PALB2), subsequently replaces RPA to form filaments on the DNA. The coating of $3^{\prime}$ overhang by Rad51 initiates the search for the repair template and invasion of homologous DNA/sister chromatid for the start of recombination process (26). When the invading strand coated with Rad51 filaments infiltrates its 
homologous partner, it results in the formation of displacement loops (D-loops). DNA polymerase delta attaches to the DNA with the help of proliferating cell nuclear antigen (PCNA) and synthesizes the missing piece of DNA. The formation of the new DNA strand results in the formation of Holliday junctions that are resolved by nickases and finally ligated to complete the HDR process restoring the original DNA sequence (27).

\section{TILTING THE REPAIR PATHWAY CHOICE IN FAVOR OF HDR FOR TARGETED TRANSGENESIS}

Depending on the nature of experimental design, the DNA repair molecular machinery can be tweaked to either favor NHEJ or HDR. In loss-of-function studies, the presence of a guide RNA that dictates Cas9 to cleave at a particular site is sufficient to induce NHEJ. Since NHEJ is error-prone, in many instances the end-product of NHEJ includes missing or added DNA sequences resulting in nonfunctional coding sequence (28). In addition, NHEJ is constitutively active in all stages of the cell cycle and is the predominant form of repair process, thus, animal or cell-based models of loss of function studies can be generated with relative ease. For these studies, the proper choice of guide RNAs to target a specific sequence and elimination of off-target effect should be of critical consideration.

HDR machinery should be utilized for "knock in" studies warranting insertion of a particular base pair or a definite stretch of DNA for enhanced therapeutic potential. However, taming endogenous cellular HDR machinery to insert a desired DNA sequence has proven to be a tricky business and more so in somatic cells (29). The problem is attributed to HDR occurring only in S/G2 phages unlike NHEJ that bears the major proportion of repair load and is active in all stages of cell cycle. Thus, the timing of DSB generation and the presence of repair template at the right time in the right location is of critical importance to create the best scenario for HDR. Generation of germ-line-based "knock-in" animal model has benefited tremendously from Cas9-based activation of HDR pathways compared to classical genome engineering with an infinitesimally small probability of incorporation of donor DNA simply based on sequence homology (30). The relative ease of HDR-mediated transgenesis in germ cells compared to somatic cells stems from the fact that during meiosis copying of the information occurs between homologous chromosomes that might contain specific sequence differences (31). Thus, for the generation of germ-line edited organisms, a piece of donor DNA with sequence homology around the cut site has reasonable possibility (albeit closer to or less than 1\%) to be attacked by a genomic $3^{\prime}$ invading strand as a repair template (32). Researchers have used this special condition to generate germ-line edited organisms from flies to pigs (33). However, the scenario is completely different in somatic cells where copying occurs from closely attached identical sister chromatid. Thus, the probability of donor DNA to be used as a repair template after DSB is extremely low in somatic cells
(34). Furthermore, in non-dividing cells, the possibility of utilizing HDR in transgenesis is tricky, due to the higher experimental barrier posed by genome editing in these types of cells (35). The major strategies that have been employed to increase HDR include chemical and genetic activation of HDR and suppression of NHEJ, stopping cells at the S/G2 stage of cell-cycle to allow more time for HDR, allowing Cas9-induced DSB only at S/G2 phase by attaching degron to Cas9 for degradation at all stages of the cell cycle where HDR is absent, enrichment of correctly edited cells with a selection marker, and increasing the concentration of donor DNA near the cut site so that the probability of the utilization of donor DNA as a repair template is increased.

\section{CHEMICAL AND GENETIC INHIBITION OF NHEJ AND ACTIVATION OF HDR}

It was previously reported that inhibition of NHEJ results in the activation of HDR as a compensatory mechanism after the generation of Cas9-mediated DSB (36). Several researchers have cleverly utilized this knowledge to inhibit NHEJ by blocking the function of key proteins involved either by using chemicals or siRNA. One of the promising studies demonstrated Scr7-mediated inhibition of NHEJ-specific LIG4 that resulted in nearly 20-fold enhancement of HDR (37). However, several follow-up studies showed that the use of Scr7 is not as promising in increasing HDR as previously described suggesting that results varied depending on the experimental systems (38). Another alternative explanation is the observation that LIG4 might not be a specific target of Scr7 (39). Nonetheless, choking other critical molecules involved in NHEJ pathway remains an attractive strategy to increase HDR. Some examples of this strategy include the use of molecules such as NU7441 (40) and Ku-0060648 (41) that inhibit DNA-PKcs. A more direct approach to increase HDR is to utilize Rad51 activator RS1 that increases CRISPR/Cas9 and TALEN-mediated knock-in efficiency (42).

Similarly, the ectopic expression of HDR molecules such as Rad51 enhances HDR (43). A recent study also demonstrated that fusing Cas9 with a domain of CtIP resulted in enhanced HDR probably by rapid positioning of the components of HDR machinery around the cut site (44). This study demonstrates that an appropriate experimental design to increase HDR should consider the optimized space and time for cutting and delivering the donor DNA.

Another approach to increase HDR is to use siRNA or shRNA to knock down key NHEJ effectors. In one such case, shRNA-mediated knockdown of Ku70, Ku80, and Lig4 individually or in combination resulted in suppression of NHEJ and 2-to-5-fold increase in HDR (37). Additional molecular players such as DNA-PK were silenced by Robert et al. using siRNA to achieve a 2-fold increase in HDR (45). Aside from chemical and siRNA/shRNA-mediated inhibition of NHEJ-specific molecules, the direct elimination of critical proteins involved 
in NHEJ pathway also increases HDR. Adenovirus serotype 4 (Ad4), E1B55K, and E4 or $\mathrm{f6}$ proteins promote the proteosomal degradation of Lig4, which results in increased $\operatorname{HDR}(37,45)$. Regulating p53 levels is another attractive strategy to increase HDR. Compared to primary cells that contain functional p53, cancer cell lines devoid of p53 activity are easier to edit genetically (46) suggesting that p53 "the guardian of the genome" is generally refractory to genome editing. To increase the efficiency of editing in primary cells, p53 activity could be conditionally decreased for a limited period of time and restored to its normal level, because constitutive loss of p53 may trigger cancer.

\section{MANIPULATING CELL CYCLE TO ENHANCE HDR IN DIVIDING CELLS}

Since HDR occurs in S/G2 phase, a straightforward strategy to increase the probability of HDR would be to ensure that all the events such as Cas9-mediated splicing at the target site occur at these stages of cell cycle. This strategy is particularly important because the continued activity of Cas9 in other stages of cell cycle increases the chances of error-prone NHEJ, basically excluding the target site from integrating the correct repair sequence. Based on this strategy, the Doudna laboratory demonstrated that G2/M synchronization of cells using nocodazole for $24 \mathrm{~h}$ followed by nucleofection of Cas9-gRNA ribonucleoprotein (RNP) and single-strand oligo deoxynucleotide (ssODN) repair template at the EMX1 locus increased HDR by a minimum of 2-fold (47). Consistent with these observations, a study conducted in human pluripotent stem cells and neural precursor cells displayed 3-to-6-fold increases in HDR when treated with nocodazole or ABT-751 (48). A recent study demonstrated that the choice of donor DNA also determines the efficiency of HDR (49). If the donor DNA is double stranded, it requires pairing with the invading genomic strand for replication by the polymerase. This process is relatively easy if the donor is already single-stranded instead of a double-stranded DNA such as plasmid. This forms the basis of single-stranded template repair (SSTR) that is mediated by Fanconi Anemia pathway and is highly efficient because no extra effort is needed to unwind the DNA (50). A slightly different strategy eliminates Cas9 at the NHEJ prone stages of cell cycle by attaching a degron that allows Cas9 only in HDR-prone stages of cell cycle. Cas9 fused with human Geminin is susceptible to proteasome-dependent degradation during G1 based on the recognition of APC-Cdh1 E3-ligase complex. Using this approach, an $87 \%$ increase in the insertion of EGFP reporter AAVS1 locus was observed in HEK-293T cells (51).

\section{MANIPULATING CELL CYCLE TO ENHANCE HDR IN NON-DIVIDING CELLS}

Transgenesis is extremely challenging in cells that are terminally differentiated because of lack of HDR activity. While studying the mechanism of HDR suppression in these cells, Orthwein et al. identified a complex molecular crosstalk between KEAP1-CUL3 E3 ubiquitin ligase complex, BRCA1, 53BP1, CtIP, BRCA2, and PALB2 (52). The investigators found that KEAP1-CUL3 complex degrades PABL2 and inhibits BRCA1 to form a complex with BRCA2-PABL2, basically excluding the possibility of RAD51 filament formation, thus no HDR. There are two other inhibitory mechanisms to suppress HDR: 53BP1-mediated inhibition of BRCA1 binding to DNA breaks and inactive CtIP (dephosphorylated) because of the lack of appropriate CDKs in G1 phase of cell cycle. In this elegant study, researchers reversed all these inhibitory brakes by ectopically expressing activated CtIP endonuclease (T847E mutant) along with the siRNA-mediated knockdown of 53BP1 and KEAP1-CUL3 complex, which resulted in the reversal of HDR repression and increased HDR in G1 cells (52).

Even though NHEJ is vilified in the world of precise genome engineering as being error-prone, it is actually an overstatement. Cas9 cleavage of target site is invariably followed by NHEJ repair, and the process is repeated until there is an error, which prevents Cas9-mediated DNA cleavage (53). Thus, the end-product of NHEJ observed might be an error, although NHEJ intrinsically does not result in such errors. This basic idea has been exploited to generate Homology-Independent Targeted Integration (HITI) and Obligate Ligation-Gated Recombination (ObLiGaRe) by utilizing NHEJ for precise gene repair in non-dividing cells. HITI is a knock-in strategy that utilizes NHEJ-based ligation of donor DNA (54). In this strategy, the donor sequence is flanked by a single circular donor or two linear donor sgRNA cleavage sites. A third identical sequence in the target locus present in the reverse orientation basically prevents reverse integration of the donor DNA.

ObLiGaRe utilizes mutant variants of Fokl domains fused to ZFNs or TALENs that induce cleavage only when paired as heterodimers but not as homodimers, and facilitates the ligation of donor DNA into the genomic cleavage site (55). In this system the target site in circular donor plasmid is identical to genomic DNA, but in reverse orientation. This strategy excludes the possibility of repeated cleavage of donor DNA after accurate insertion.

\section{PHYSICALLY POSITIONING DONOR DNA NEAR THE CLEAVAGE SITE}

After the 3'SS DNA is coated with Rad51, the homologous DNA sequence is used as a repair template (32). In somatic cells, the copying occurs from the sister chromatid whereas in germ cells it starts in the homologous chromosome. By supplying the donor DNA with homology-arms around the target site, a researcher can hope that the donor DNA externally supplied is used as a repair template. Since the probability of invading genomic 3'SS DNA to attack donor 
DNA is very low, several recent studies have positioned donor DNA near the target site instead of randomly floating them in the nucleoplasm and this strategy has been successful to increase the HDR efficiency. Following this theory, a recent report demonstrated that the fusion of Cas9 with the donor DNA using SNAP-tag resulted in a 24-fold increase in HDR (56). A similar study conjugated donor DNA with Cas9 via biotin-mono-avidin resulted in 2-to-5-fold increase in knock-in mice generation (57).

A recent study performed the localization of donor DNA near the target site using biotin-avidin conjugation to another level by delivering the components of genome editing (streptavidin Cas9, guide RNA, and biotinylated donor DNA) in the two-cell stage of mouse development, the stage at which G2 phase is the longest, and demonstrated increased HDR in most of the loci tested (58). Collectively, increasing the local concentration of donor DNA at the cut site increases the probability of use as a repair template, hence increase in HDR frequency.

\section{POSITIVE SELECTION AND ENRICHMENT OF EDITED CELLS}

Another approach to obtain higher percentage of cells that have undergone correct HDR entails a classical gene targeting strategy: to enrich the cells by positively selecting them via insertion of an antibiotic resistance gene or any selectable marker, and landing them in a safe-harbor locus such as AAVS1 (59). However, because of the number of cells that undergo precise editing is much higher in CRISPR/Cas, the process is less intense than classical recombineering for screening positively edited cells. This approach is more appropriate for experimental than therapeutic purpose because of the presence of selection markers in the final edit.

Several strategies have been implemented to increase the efficiency of HDR in general and with some urgency in somatic cells. Since germline editing raises ethical concerns associated, our ability to rewrite the erroneous genetic code in somatic cells with the correct genetic information holds enormous therapeutic potential. Elegant studies conducted within a span of several years have pinpointed several criteria such as inhibition of NHEJ, activation of HDR, controlled editing in HDR permissive stages of cell cycle, and increasing local concentration of donor DNA near the target cleavage site as playing an important role to boost HDR in somatic cells. Further studies should focus on finding the optimum combination of conditions that is non-lethal in order to maximize HDR.

\section{CONCLUSIONS AND FUTURE CHALLENGES}

Several elegant studies have pinpointed the potential shortcomings of CRISPR-Cas9-mediated genome-engineering technology in general, and more specifically for therapeutic applications. In addition to the lower efficiency of HDR in CRISPR-Cas-mediated transgenesis, three critical challenges need to be addressed to ensure a giant transition of this technology from 'bench to bed'. The first challenge is the immune reaction that is already activated in humans against the most widely used forms of Cas9. A recent study demonstrated that majority of humans have already developed immune reaction against Cas9 from Staphyloccous aureus (SaCas9) and Streptococcus pyogenes (SpCas9) (60). Thus, any form of gene therapy that uses SpCas9 or SaCas9 (or any other Cas proteins from microorganisms that have regular contact with human beings) will probably be rejected by the human immune system. The solution is to use a recoded version of Cas protein, which evades the immune system. Another strategy is to identify and use Cas proteins from microorganisms that have not been in physical contact with human beings, such as those derived from deep oceans or volcanic sites. The second critical issue is regarding the role of p53 after the generation of DSB. Being the 'guardian of the genome', p53 is activated in response to DNA damage whether it is induced under natural circumstances or experimentally by Cas9. Compared to primary cells that retain functional p53, cancer cell lines that lack p53 function are amenable to genome editing (46). This observation has led to many researchers speculating whether the therapeutic cells that contain the corrected sequence after gene therapy are actually compromised in p53 function and cause cancer? Therefore, it is essential to fully sequence and validate therapeutic cells for p53 function before application in humans. The third critical issue relates to the off-target activity of Cas 9 and the possibility of abnormal DNA rearrangement after DSB. Almost all of the studies investigating Cas9 activity characterize the nature of $\mathrm{NHEJ} / \mathrm{HDR}$ by sequencing the narrow region around the cleavage site to measure the on-target activity, and random regions in the genome to examine off-target activity (61). However, the strategy is limited by the inability of PCR to detect the big chunk of DNA or the whole chromosome missing after the generation of DSB. The presence of undetectable and undesired mutations in cells targeted for gene therapy will have devastating consequences for the patient, and more generally adversely affect the reputation of the field. To address this issue, Allan Bradley's group recently undertook a bold study to scan large chunks of the genome after Cas9-induced DNA damage (62). The group observed that repair of double-strand breaks induced by CRISPR-Cas9 leads to large deletions and complex rearrangements (62). This study demonstrates the need for careful scrutiny of the overhyped technology before human application. Rapid screening methods must be developed to scan the total genome for the presence of undesired mutations that are induced by CRISPR-Cas9.

Even though 6 years is a relatively short time frame to judge the success of a technology, there is no doubt that CRISPR/Cas system has revolutionized genome engineering. As it is with 
every promising technology in the history of mankind, the hope CRISPR-Cas9 has generated must be matched with rigorous quality control experiments to increase its efficiency and reduce side effects before its potential application in gene therapy can be fully realized.

\section{CONFLICTS OF INTEREST}

The authors have no conflicting interests.

\section{REFERENCES}

1. Smith HO and Wilcox KW (1992) A restriction enzyme from Hemophilus influenzae. I. Purification and general properties. 1970. Biotechnology 24, 38-50

2. Capecchi MR (1989) Altering the genome by homologous recombination. Science 244, 1288-1292

3. Smithies O, Gregg RG, Boggs SS, Koralewski MA and Kucherlapati RS (1985) Insertion of DNA sequences into the human chromosomal beta-globin locus by homologous recombination. Nature 317, 230-234

4. Thomas KR, Folger KR and Capecchi MR (1986) High frequency targeting of genes to specific sites in the mammalian genome. Cell 44, 419-428

5. Watts G (2007) Nobel prize is awarded for work leading to "knockout mouse". BMJ 335, 740

6. Lin FL, Sperle K and Sternberg N (1985) Recombination in mouse $\mathrm{L}$ cells between DNA introduced into cells and homologous chromosomal sequences. Proc Natl Acad Sci U S A 82, 1391-1395

7. Rouet P, Smih F and Jasin M (1994) Introduction of double-strand breaks into the genome of mouse cells by expression of a rare-cutting endonuclease. Mol Cell Biol 14, 8096-8106

8. Kim YG, Cha J and Chandrasegaran S (1996) Hybrid restriction enzymes: zinc finger fusions to Fok I cleavage domain. Proc Natl Acad Sci U S A 93, 1156-1160

9. Boch J, Scholze H, Schornack S et al (2009) Breaking the code of DNA binding specificity of TAL-type III effectors. Science 326, 1509-1512

10. Zhang F, Cong L, Lodato S, Kosuri S, Church GM and Arlotta P (2011) Efficient construction of sequence-specific TAL effectors for modulating mammalian transcription. Nat Biotechnol 29, 149-153

11. Gupta RM and Musunuru K (2014) Expanding the genetic editing tool kit: ZFNs, TALENs, and CRISPR-Cas9. J Clin Invest 124, 4154-4161

12. Jinek M, Chylinski K, Fonfara I, Hauer M, Doudna JA and Charpentier E (2012) A programmable dual-RNA-guided DNA endonuclease in adaptive bacterial immunity. Science 337, 816-821

13. Mali P, Yang L, Esvelt KM et al (2013) RNA-guided human genome engineering via Cas 9. Science 339, 823-826

14. Adli M (2018) The CRISPR tool kit for genome editing and beyond. Nat Commun 9, 1911

15. Rothkamm K, Kruger I, Thompson LH and Lobrich M (2003) Pathways of DNA double-strand break repair during the mammalian cell cycle. Mol Cell Biol 23, 5706-5715
16. Mao Z, Bozzella M, Seluanov A and Gorbunova V (2008) DNA repair by nonhomologous end joining and homologous recombination during cell cycle in human cells. Cell Cycle 7, 2902-2906

17. Davis AJ and Chen DJ (2013) DNA double strand break repair via non-homologous end-joining. Transl Cancer Res 2, 130-143

18. Chang $\mathrm{HH}$ and Lieber MR (2016) Structure-Specific nuclease activities of Artemis and the Artemis: DNA-PKcs complex. Nucleic Acids Res 44, 4991-4997

19. Her J and Bunting SF (2018) How cells ensure correct repair of DNA double-strand breaks. J Biol Chem 293, 10502-10511

20. Bennardo N, Cheng A, Huang N and Stark JM (2008) Alternative-NHEJ is a mechanistically distinct pathway of mammalian chromosome break repair. PLoS Genet 4 e1000110

21. Chiruvella KK, Liang Z and Wilson TE (2013) Repair of double-strand breaks by end joining. Cold Spring Harb Perspect Biol 5, a012757

22. Salsman J and Dellaire G (2017) Precision genome editing in the CRISPR era. Biochem Cell Biol 95, 187-201

23. Branzei D and Foiani M (2008) Regulation of DNA repair throughout the cell cycle. Nat Rev Mol Cell Biol 9, 297-308

24. Marechal A and Zou L (2013) DNA damage sensing by the ATM and ATR kinases. Cold Spring Harb Perspect Biol 5, 1-17

25. Symington LS (2014) End resection at double-strand breaks: mechanism and regulation. Cold Spring Harb Perspect Biol 6, 1-18

26. Buisson R, Niraj J, Pauty J et al (2014) Breast cancer proteins PALB2 and BRCA2 stimulate polymerase eta in recombination-associated DNA synthesis at blocked replication forks. Cell Rep 6, 553-564

27. Jasin M and Rothstein R (2013) Repair of strand breaks by homologous recombination. Cold Spring Harb Perspect Biol 5, a012740

28. Hug N, Longman D and Caceres JF (2016) Mechanism and regulation of the nonsense-mediated decay pathway. Nucleic Acids Res 44, 1483-1495

29. Carroll D (2016) Genome editing: progress and challenges for medical applications. Genome Med 8, 120

30. Port F, Chen HM, Lee T and Bullock SL (2014) Optimized CRISPR/Cas tools for efficient germline and somatic genome engineering in Drosophila. Proc Natl Acad Sci U S A 111, E2967-2976

31. Sybenga J (1999) What makes homologous chromosomes find each other in meiosis? A review and an hypothesis. Chromosoma 108, 209-219

32. Ma CJ, Gibb B, Kwon Y, Sung P and Greene EC (2017) Protein dynamics of human RPA and RAD51 on ssDNA during assembly and disassembly of the RAD51 filament. Nucleic Acids Res 45, 749-761

33. Ruan J, Xu J, Chen-Tsai RY and Li K (2017) Genome editing in livestock: Are we ready for a revolution in animal breeding industry? Transgenic Res 26, 715-726

34. Taleei R and Nikjoo H (2013) Biochemical DSB-repair model for mammalian cells in G1 and early $S$ phases of the cell cycle. Mutat Res 756, 206-212 
35. Nami F, Basiri M, Satarian L, Curtiss C, Baharvand $H$ and Verfaillie C (2018) Strategies for In Vivo Genome Editing in Nondividing Cells. Trends Biotechnol 36, 770-786

36. Maruyama T, Dougan SK, Truttmann MC, Bilate AM, Ingram JR and Ploegh HL (2015) Increasing the efficiency of precise genome editing with CRISPR-Cas9 by inhibition of nonhomologous end joining. Nat Biotechnol 33, 538-542

37. Chu VT, Weber T, Wefers B et al (2015) Increasing the efficiency of homology-directed repair for CRISPR -Cas9-induced precise gene editing in mammalian cells. Nat Biotechnol 33, 543-548

38. Pinder J, Salsman J and Dellaire G (2015) Nuclear domain 'knock-in' screen for the evaluation and identification of small molecule enhancers of CRISPR-based genome editing. Nucleic Acids Res 43, 9379-9392

39. Greco GE, Matsumoto Y, Brooks RC, Lu Z, Lieber MR and Tomkinson AE (2016) SCR7 is neither a selective nor a potent inhibitor of human DNA ligase IV. DNA Repair (Amst) 43, 18-23

40. Leahy JJ, Golding BT, Griffin RJ et al (2004) Identification of a highly potent and selective DNA-dependent protein kinase (DNA-PK) inhibitor (NU7441) by screening of chromenone libraries. Bioorg Med Chem Lett 14, 6083-6087

41. Munck JM, Batey MA, Zhao $Y$ et al (2012) Chemosensitization of cancer cells by KU-0060648, a dual inhibitor of DNA-PK and PI-3K. Mol Cancer Ther 11, 1789-1798

42. Song J, Yang D, Xu J, Zhu T, Chen YE and Zhang J (2016) RS-1 enhances CRISPR/Cas9- and TALEN-mediated knock-in efficiency. Nat Commun 7, 10548

43. Yu S, Song Z, Luo J, Dai Y nd Li N (2011) Over-expression of RAD51 or RAD54 but not RAD51/4 enhances extrachromosomal homologous recombination in the human sarcoma (HT-1080) cell line. J Biotechnol 154, 21-24

44. Charpentier M, Khedher AHY, Menoret S et al (2018) CtIP fusion to Cas9 enhances transgene integration by homology-dependent repair. Nat Commun 9, 1133

45. Robert F, Barbeau M, Ethier S, Dostie J and Pelletier J (2015) Pharmacological inhibition of DNA-PK stimulates Cas9-mediated genome editing. Genome Med 7, 93

46. Haapaniemi E, Botla S, Persson J, Schmierer B and Taipale J (2018) CRISPR-Cas9 genome editing induces a p53-mediated DNA damage response. Nat Med 24, 927-930

47. Lin S, Staahl BT, Alla RK and Doudna JA (2014) Enhanced homology-directed human genome engineering by controlled timing of CRISPR/Cas9 delivery. Elife 3, e04766

48. Yang D, Scavuzzo MA, Chmielowiec J, Sharp R, Bajic A and Borowiak M (2016) Enrichment of G2/M cell cycle phase in human pluripotent stem cells enhances HDR-mediated gene repair with customizable endonucleases. Sci Rep 6, 21264
49. Song F and Stieger K (2017) Optimizing the DNA Donor Template for Homology-Directed Repair of Double-Strand Breaks. Mol Ther Nucleic Acids 7, 53-60

50. Richardson CD, Kazane KR, Feng SJ et al (2018) CRISPR-Cas9 genome editing in human cells works via the Fanconi Anemia pathway. Nat Genetics 50, 1132-1139

51. Gutschner T, Haemmerle M, Genovese G, Draetta GF and Chin L (2016) Post-translational Regulation of Cas9 during G1 Enhances Homology-Directed Repair. Cell Rep 14, 1555-1566

52. Orthwein A, Noordermeer SM, Wilson MD et al (2015) A mechanism for the suppression of homologous recombination in G1 cells. Nature 528, 422-426

53. Zaboikin $\mathrm{M}$, Zaboikina $\mathrm{T}$, Freter $\mathrm{C}$ and Srinivasakumar $\mathrm{N}$ (2017) Non-Homologous end joining and homology directed DNA repair frequency of double-stranded breaks introduced by genome editing reagents. PLoS One 12, e0169931

54. Suzuki K, Tsunekawa Y, Hernandez-Benitez R et al (2016) In vivo genome editing via CRISPR/Cas9 mediated homology-independent targeted integration. Nature 540, 144-149

55. Maresca M, Lin VG, Guo N and Yang Y (2013) Obligate ligation-gated recombination (ObLiGaRe): custom-designed nuclease-mediated targeted integration through nonhomologous end joining. Genome Res 23, 539-546

56. Savic N, Ringnalda FC, Lindsay $\mathrm{H}$ et al (2018) Covalent linkage of the DNA repair template to the CRISPR-Cas9 nuclease enhances homology- directed repair. Elife 7, 1-18

57. Ma M, Zhuang F, Hu X et al (2017) Efficient generation of mice carrying homozygous double-floxp alleles using the Cas9-Avidin/Biotin-donor DNA system. Cell Res 27, 578-581

58. Gu B, Posfai E and Rossant J (2018) Efficient generation of targeted large insertions by microinjection into two-cellstage mouse embryos. Nat Biotechnol 36, 632-637

59. Oceguera-Yanez F, Kim SI, Matsumoto T et al (2016) Engineering the AAVS1 locus for consistent and scalable transgene expression in human iPSCs and their differentiated derivatives. Methods 101, 43-55

60. Charlesworth CT, Deshpande PS, Dever DP et al (2018) Identification of Pre-Existing Adaptive Immunity to Cas9 Proteins in Humans. bioRxiv https://doi.org/10.1101/24 3345

61. Biehs R, Steinlage M, Barton O et al (2017) DNA Doublestrand break resection occurs during non-homologous end joining in G1 but Is distinct from resection during homologous recombination. Mol Cell 65, 671-684 e675

62. Kosicki M, Tomberg K and Bradley A (2018) Repair of double-strand breaks induced by CRISPR-Cas9 leads to large deletions and complex rearrangements. Nat Biotechnol 36, 765-771 